\title{
UZH Hosts Second Dorothy Crowfoot Hodgkin Symposium and Awards First Hoffmann Centennial Medal
}

\author{
Nathaniel S. Finney*
}

Abstract: On Friday, April 27th, the Organic Chemistry Institute (OCl) hosted the second Dorothy Crowfoot Hodgkin Symposium at the University of Zurich, as well as the presentation of the Hoffmann Centennial Medal for the Study of Natural Products. Organized by Prof. Kim Baldridge, the annual DCH symposium commemorates the remarkable scientific and social legacy of Dorothy Crowfoot Hodgkin (See http://www.oci.unizh.ch/diversa/ dch/2007/ for further information.)

Keywords: Dorothy Crowfoot Hodgkin Symposium · Hoffmann Centennial Medal

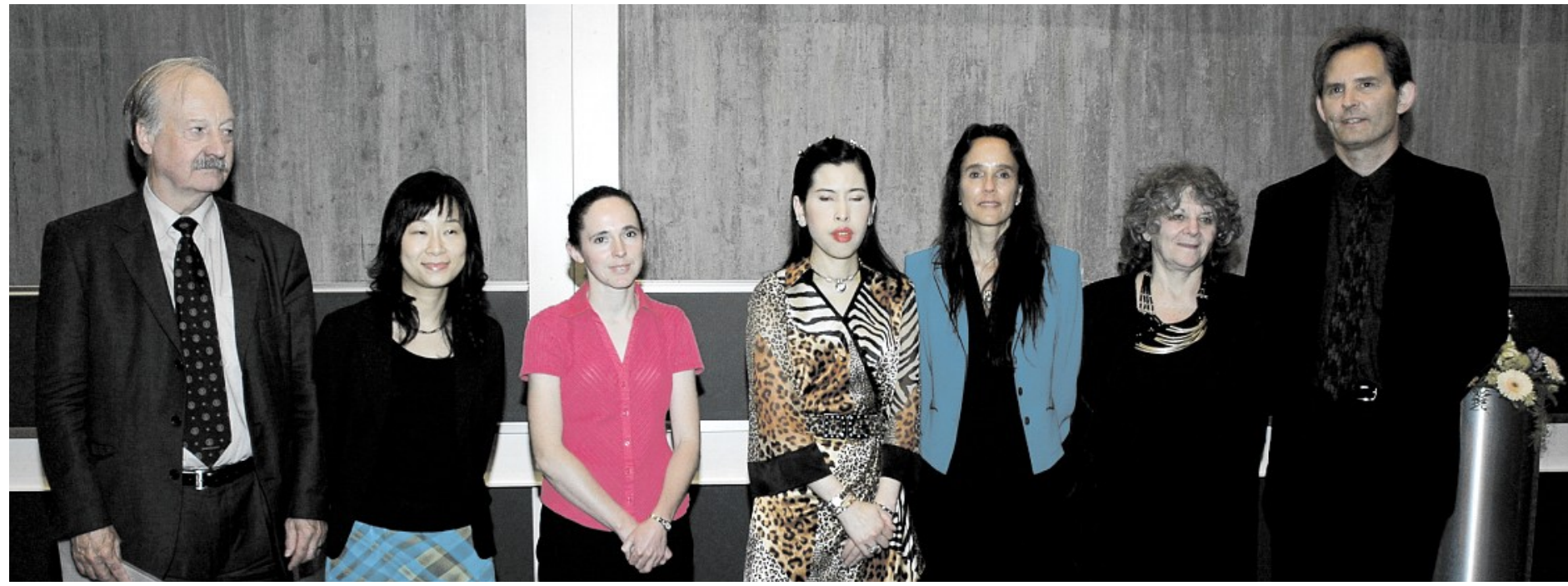

Participants in the second Dorothy Crowfoot Hodgkin Symposium (left to right): UZH President Prof. Hans Weder, Prof. Pauline Chiu (University of Hong Kong), Dr. Debra Wallace (Merck Process UK), HRH Princess Chulabhorn of Thailand (Chulabhorn Research Institute), Prof. Kim Baldridge (UZH), Prof. Ada Yonath (Weizmann Institute), Director of the OCI Prof. Jay Siegel.

${ }^{*}$ Correspondence: PD Dr. N. S. Finney

Institute of Organic Chemistry

University of Zurich UZH

Winterthurerstrasse 190

$\mathrm{CH}-8057$ Zurich

E-Mail: finney@oci.uzh.ch
The first speaker of the Symposium was Dr. Debra Wallace of Merck Process, UK. Dr. Wallace described recent advances in the synthesis of Taranabant, a CB1R 'inverse agonist' currently in Phase III clinical trials as an appetite suppressant for the treatment of obesity. Taranabant is a secondary acylated amine with two stereocenters and three substituted aromatic rings. The medicinal chemistry synthesis allowed for the initial preparation of material but was unsuitable as a manufacturing process, as it involved several aryl stannane intermediates, the use of an alkyl azide, chromatography at almost every step and a late-stage resolution of racemic material. Dr. Wallace described the strategic thinking behind the decision to address removal of the chromatography and azide and tin chemistry first, enabling clean, scalable access to intermediates which could then be investigated for resolution or enantioselective transformation. After exploring several promising but ultimately unsatisfactory routes, a highly selective route based on enantioselective hydrogenation was developed - the discovery of which highlighted the importance of Merck's internal high-throughput catalyst screening group. 
The second lecture was delivered by Prof. Pauline Chiu of the University of Hong Kong. Prof. Chiu described her group's recent total synthesis of pseudolaric acid A. The pseudolaric acids are isolated from a rare tree, the bark of which has long been used in traditional Chinese medicine. The pseudolaric acids exhibit a broad range of biological activities, the most notable of which is the efficacy of pseudolaric acid B $(\mathrm{paB})$ in treating Taxol-resistant liver cancer in nude mice. While much remains unknown, it appears that paB functions in part by simultaneously inhibiting tubulin polymerization and avoiding an efflux pump commonly associated with multi-drug resistance. Once again emphasizing the power of catalytic enantioselective transformations, Prof. Chiu's elegant and efficient approach to the complex tricyclic core of the pseudolaric acids began with the assembly of an acyclic precursor via a catalytic enantioselective aldol condensation with a pyruvate ester. After subsequent elaboration to form an $\alpha$-diazo-1,4-diketone, exposure to a chiral $\mathrm{Rh}$ (I) catalyst led to the formation of cyclic ylide which underwent enantioselective dipolar cycloaddition to form the key [5.3.0] ring system. A short series of transformations then provided pseudolaric acid A. While further synthetic studies are ongoing, the current route already allows the synthesis of derivatives, notably those for photochemical cross-linking that should reveal the natural protein target(s) of the pseudolaric acids.

In a dramatic shift of length scales, Prof. Ada Yonath of the Weizmann Institute next provided an overview of the structure and function of the ribosome, with particular emphasis on recent advances in molecular-level mechanistic understanding. Connecting the ribosome to drugs and natural products, Prof. Yonath noted that $\sim 40 \%$ of all known antibiotics target protein biosynthesis, a process that consumes almost $80 \%$ of the metabolic energy of log-phase bacteria. As molecular understanding of ribosome structure has advanced (in large part due to Prof. Yonath's work), clear differences between bacterial and human ribosomal structure have emerged, and the bacterial ribosome thus remains a very promising drug target. Expanding on recent mechanistic work, Prof. Yonath drew attention to the surprising - and only recently identified - pseudo- $C_{2}$-symmetry of the $\mathrm{A}$ and $\mathrm{P}$ sites in the large ribosomal subunit. Despite the absence of sequence homology, this pseudo-symmetry appears to play an important functional role in peptide bond formation, which takes place at the interface of these two sites. Recent studies with an acceptor stem mimic (a truncated version

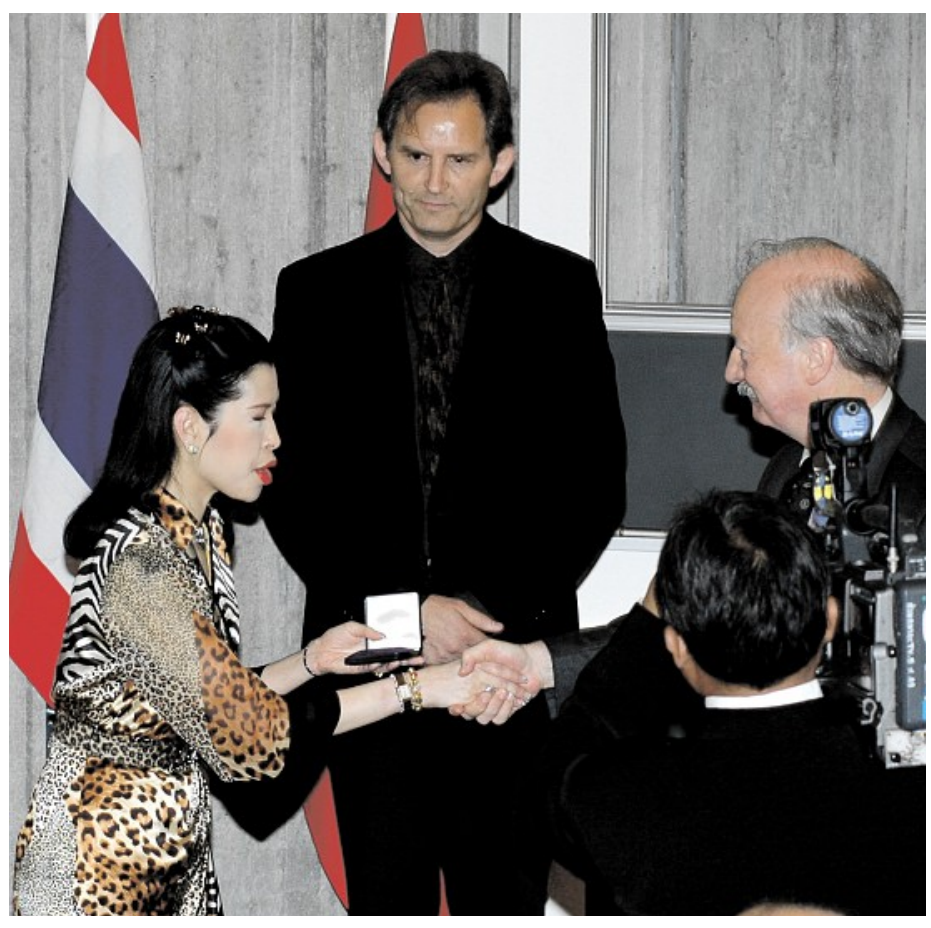

HRH Princess Chulabhorn receives the first Hoffmann Award for the Study of Natural Products from UZH President Weder

of tRNA that still possesses the amino-acid bearing tail) suggest that incorporation of a new amino acid in a growing peptide chain occurs via a mechanism in which twisting of the acceptor stem is coupled to simultaneous peptide bond formation and extrusion of the growing protein, rather than by a stepwise mechanism. The potential gating role of a key active site exo-nucleotide, supported by kinetic, crystallographic and computational study, was also discussed.

Following a brief retrospective of the accomplishments of Albert Hoffmann, the first Hoffmann Centennial Medal for the Study of Natural Products was bestowed on Her Royal Highness Princess Chulabhorn of Thailand. Founder of the Chulabhorn Research Institute, Princess Chulabhorn has long been dedicated to furthering human welfare through chemical research, particularly as it relates to natural products and the environment. The talk delivered by the Princess provided a broad overview of the unique biodiversity of Thailand, illustrated by several case studies in the isolation, characterization and biological evaluation of new natural products. Of particular interest were the flavonoid lupinofolin, which exhibits activity against the P-388 leukemia cell line, and andrographolide, a terpenoid that inhibits platelet aggregation. Observing that $>60 \%$ of known anticancer agents and $>75 \%$ of known anti-infectives derive from natural sources, the Princess strongly emphasized the importance of natural product isolation as a source of new medicines, and exhorted the global pharma- ceutical industry to revitalize efforts in this important discipline.

The Symposium was followed by a poster session that included more than 40 participants from numerous European universities. The OCI would like to express its appreciation to Merck, Swiss Re and the Laboratory for Process Research for generous financial support, and the local chemistry community for its enthusiastic participation. Additional OCI-hosted events in 2007 will include the Karrer Medallist Lecture (Prof. Steven Ley, Cambridge Univ.) on June 20th. See http://www.oci.unizh.ch/diversa/Karrer2007/index.html for details.

Received: May 9, 2007 